DOI 10.35381/cm.v7i1.490

\title{
Evaluación de la productividad del talento humano basado en un modelo de competencias laborales
}

\section{Assessing human talent productivity based on a work skills model}

\author{
Willians Antonio Guerrero-Castro \\ willguerrero1512@gmail.com \\ Universidad Politécnica Territorial Alonzo Gamero, Santa Ana de Coro \\ Venezuela \\ https://orcid.org/0000-0003-2193-0728 \\ Rously Eedyah Atencio-González \\ uq.rouslyatenio@uniandes.edu.ec \\ Universidad Regional Autónoma de los Andes, Quevedo \\ Ecuador \\ https://orcid.org/0000-0001-6845-1631 \\ Jesús Antonio Pirona \\ ipirona287@gmail.com \\ Instituto de Investigación y Estudios Avanzados Koinonía, Santa Ana de Coro \\ Venezuela \\ https://orcid.org/0000-0001-9811-8047
}

Recibido: 10 de marzo de 2021 Aprobado: 15 de junio de 2021 


\title{
RESUMEN
}

La evaluación de competencias laborales es un proceso complejo, que requiere como pasos previos la definición de perfiles ocupacionales, estructurados en torno a conocimientos, habilidades, conductas individuales y sociales en el trabajo. La investigación, desde el paradigma cuantitativo, se desarrolló dentro de los parámetros de un tipo de investigación de campo que, consistió en la recolección de datos directamente de la realidad donde ocurren los hechos sin manipular o controlar variables. Cuyo objetivo fue diagnosticar la situación actual en cuanto a la evaluación del desempeño llevada a cabo al personal administrativo de las instituciones universitarias. Para la obtención de los datos se utilizó un cuestionario, destacando la importante la aplicación de la medición. De acuerdo a los resultados obtenidos, se concluye que se hace necesario aplicar un instrumento de evaluación basado en competencias laborales que sustituya el modelo aplicado hasta el momento, basado en resultados por objetivos de desempeño individual.

Descriptores: Apreciación de la actuación; habilidad; desarrollo de recursos humanos; actitud laboral; comportamiento. (Palabras tomadas del Tesauro UNESCO).

\begin{abstract}
The evaluation of labor competencies is a complex process, which requires as previous steps the definition of occupational profiles, structured around knowledge, skills, individual and social behaviors at work. This research from the quantitative paradigm was developed within the parameters of a type of field research that consisted of collecting data directly from the reality where the events occur without manipulating or controlling variables. Whose objective was to diagnose the current situation regarding the performance evaluation carried out to the administrative personnel of the university institutions. To obtain the data, a questionnaire was used, highlighting the important application of the measurement. According to the results obtained, it is concluded that it is necessary to apply an evaluation instrument based on labor competencies that replaces the model applied so far, based on results for individual performance objectives.
\end{abstract}

Descriptors: Performance appreciation; ability; human resources development; work attitude; behavior. (Words taken from the UNESCO Thesaurus). 


\section{INTRODUCCIÓN}

La administración de recursos humanos, en la actualidad, se desenvuelve en un ambiente competitivo y globalizado por lo que las organizaciones dependerán de lo eficaz y eficiente en que estos recursos funcionen. La infraestructura, tecnología, recursos financieros y materiales son solo elementos físicos que necesitan ser administrados correctamente y con inteligencia. A diferencia de hace unas décadas donde lo principal de las organizaciones lo constituía la producción, dejando a un lado los requerimientos, las necesidades y las atenciones básicas laborales del personal trabajador.

Por ende, las personas son el único factor dinámico de las organizaciones, sean privadas o públicas, de bienes o servicios, lucrativas o no. La calidad de los funcionarios y trabajadores en el área administrativa que se desempeñan en un organismo público debe estar orientada hacia la excelencia, ampliando los conocimientos y habilidades, para un óptimo desenvolvimiento. Evaluar a los trabajadores en las organizaciones es tremendamente importante y muy difícil de lograr de manera adecuada. En efecto, es un proceso sistémico, muy caro y lleva bastante tiempo construirlo y afinarlo, por lo tanto, la organización debe asignarle el tiempo suficiente y la importancia que requiere; afortunadamente, la mayoría de las organizaciones están tomando consciencia de ello, y ya casi no se encuentran empresas públicas ni privadas que sean ajenas a esta realidad. (Sánchez-Henríquez \& Calderón-Calderón, 2012:59)

Sin embargo, las instituciones públicas presentan grandes adversidades de gestión en materia laboral por su destacado crecimiento del recurso humano, esto trae como consecuencia que el Estado Nacional se le dificulte satisfacer las necesidades de todos los trabajadores que desempeñan funciones en estos organismos. Los gerentes se desenvuelven en un ambiente cambiante, con deficiencias presupuestarias, que obstaculizan el desarrollo de la gestión administrativa. Específicamente en la educación universitaria, la administración de recursos humanos ha ocupado un lugar primordial, 
entendiendo que la productividad dependerá de los logros en términos de eficacia y eficiencia hacia el logro de los objetivos señalados.

En este sentido, el desempeño laboral es el rendimiento global del empleado, en algunos casos parte de los empleados procura obtener retroalimentación sobre la manera en que cumplen sus actividades, por otro lado, las personas que tienen a su cargo la dirección de otros empleados deben evaluar el desempeño individual para decidir las acciones que se deben considerar para alcanzar las metas propuestas. Las evaluaciones informales, basadas en el trabajo diario, son necesarias pero insuficientes. Por tanto, si se cuenta con un sistema formal y sistemático de retroalimentación, el departamento de personal puede identificar a los empleados que cumplen o exceden lo esperado y a los que no lo hacen. Asimismo, ayuda a evaluar los procedimientos de reclutamiento, selección y orientación e incluso las decisiones sobre promociones internas, compensaciones y otras más del área del departamento de personal, que dependen de la información sistemática y bien documentada disponible sobre el empleado. No obstante, no basta con cancelar el salario por el servicio prestado hacia la institución, los trabajadores necesitan de un incentivo, una recompensa, basada en la cuantificación de su aporte por realizar sus labores de una manera eficaz y eficiente en competitividad sana con otros trabajadores, dicho estímulo adicional lo constituyó en sus inicios la medición de su Evaluación de Desempeño Individual (ODI) el cual ha perdido su finalidad al desvirtuarse sus resultados obtenidos, a través de lo largo del tiempo de su aplicación.

La función pública está constituida por el conjunto de arreglos institucionales mediante los cuales se articulan y gestiona el empleo público y las personas que lo integran en una realidad nacional. Comprende diversas normas escritas o informales, estructuras, pautas culturales, políticas explícitas o implícitas, procesos, prácticas y actividades cuya finalidad es garantizar un manejo adecuado de los recursos humanos (rrhh), en el marco de una administración pública profesional y eficaz (Carta Iberoamericana de la Función Pública, 2003). Además, necesita de instrumentos de planificación, mediante el cual la 
organización realiza el estudio cuantitativo y cualitativo de sus necesidades en materia de recursos humanos a corto, mediano y largo plazo, contrastando las necesidades detectadas con sus capacidades internas e identificando las acciones que deben emprenderse para cubrir las diferencias. La planificación constituye el nexo obligado entre la estrategia organizativa y el conjunto de políticas y prácticas de gestión del empleo y las personas (Centro Latinoamericano de Administración para el Desarrollo, 1972).

Para lograr una planificación de calidad, será necesario disponer de sistemas de información sobre el trabajador que sean capaces de permitir un conocimiento real y actualizado de las disponibilidades cuantitativas y cualitativas de recursos humanos, en la actualidad denominado talento humano. Así será posible:

- La optimización de los efectivos cuantitativos y cualitativos disponibles.

- La adaptación flexible de las políticas y prácticas de personal a los cambios producidos en la organización.

- La correcta distribución de los recursos disponibles y la redistribución flexible de las personas en función de las necesidades organizativas.

- Seguimiento y actualización de sus previsiones.

- La participación activa de los directivos en los procesos de planificación.

En efecto la evaluación de competencias laborales es un proceso complejo, que requiere como pasos previos la definición de perfiles ocupacionales, estructurados en torno a conocimientos, habilidades, conductas individuales y sociales en el trabajo, debe formar parte de las políticas de gestión de recursos humanos incorporados por todo sistema de servicio. Disponer de sistemas formales de evaluación de la productividad permite: obtener informaciones necesarias para adoptar decisiones en diferentes áreas de la gestión de los trabajadores, validar políticas y prácticas de gestión de recursos humanos, contrastando y valorando su impacto sobre el comportamiento humano en el trabajo, 
orientar el desarrollo de los trabajadores y su crecimiento profesional y mejorar la motivación y el rendimiento de las personas en el puesto de trabajo.

Para tal efecto la evaluación de la productividad o de las competencias laborales es un procedimiento estructural y sistemático para medir, evaluar e influir sobre los atributos, comportamientos y resultados relacionados con el trabajo, así como el grado de absentismo, con el fin de descubrir en qué medida es productivo el empleado y si podrá mejorar su rendimiento futuro (Rodríguez-Serrano, 2004). De allí se desprende la necesidad de lograr una sinapsis envolvente entre los objetivos organizacionales ya definidos en las empresas e instituciones y las labores de los trabajadores en función de sus competencias para alcanzarlos.

Es a partir de la Ley del Estatuto de la Función Pública (LEFP), creada en Gaceta Oficial No. 37.522 de fecha 06 de septiembre de 2002, que se le otorga el carácter de obligatoriedad para toda la administración pública de aplicar una evaluación de desempeño la cual establece en el Capítulo IV, "Evaluación del Desempeño", lo siguiente: Artículo 57. La evaluación de los funcionarios y funcionarias públicos en los órganos y entes de la Administración Pública comprenderá el conjunto de normas y procedimientos tendentes a evaluar su desempeño. Los órganos y entes de la Administración Pública Nacional deberán presentar al Ministerio de Planificación y Desarrollo, para su aprobación, los resultados de sus evaluaciones, como soporte de los movimientos de personal que pretendan realizar en el próximo año fiscal y su incidencia en la nómina del personal activo, conjuntamente con el plan de personal, determinando los objetivos que se estiman cumplir durante el referido ejercicio fiscal.

Asimismo, en su articulado № 58, establece que: la evaluación deberá ser realizada dos veces por año sobre la base de los registros continuos de actuación que debe llevar cada supervisor. En el proceso de evaluación, el funcionario deberá conocer los objetivos del desempeño a evaluar, los cuales serán acordes con las funciones inherentes al cargo. De aquí resalta que, al hablar de evaluación de la productividad, se hace énfasis al 


\section{CIENCIAMATRIA}

Revista Interdisciplinaria de Humanidades, Educación, Ciencia y Tecnología

Año VII. Vol. VII. N¹. Edición Especial. 2021

Hecho el depósito de ley: pp201602FA4721

ISSN-L: 2542-3029; ISSN: 2610-802X

Universidad Nacional Experimental Francisco de Miranda (UNEFM). Santa Ana de Coro. Venezuela

Willians Antonio Guerrero-Castro; Rously Eedyah Atencio-González; Jesús Antonio Pirona

personal administrativo, con relación laboral permanente o no, dentro de los órganos y entes de la Administración Pública Nacional.

Es fundamental tener en cuenta que una evaluación muy negativa, obviamente, tiene un efecto devastador en la moral del empleado. De hecho, incluso evaluaciones positivas, pero no tan positivas como la persona espera, tiene un efecto negativo en su moral. Aparece la dificultad principal: la mayoría de las personas creen que trabaja muy bien (incluso si no lo hacen), es simplemente un truco que juega la mente para hacer soportable la existencia y permitir juntar fuerzas para levantarnos todas las mañanas para ir a la oficina a cumplir con sus labores. $Y$ aquellos que realmente trabajan bien, esperarán un resultado extraordinario en todos los puntos, cosa que rara vez sucede porque, de lo contrario, no existirían nuevos aspectos por mejorar.

En cuanto a las necesidades que las empresas enfrentan ante las demandas de los cambios tecnológicos y la globalización, así como de la mayor exigencia por parte de los usuarios de sus servicios, requieren de una mejor identificación de las habilidades y capacidades de los trabajadores que se desempeñarán en las mismas (trabajadores competentes en sus cargos). Tomando en consideración lo antes expuesto se podrá considerar la investigación a partir de la siguiente interrogante: ¿Será necesario que los factores a evaluar en el desempeño estén en relación directa con las competencias de las personas, ya que no se consigue estímulos de motivación utilizando factores generales por objetivos?

De acuerdo a la afirmación de Freitas (2007: 23), los individuos ocupan un puesto o cargo, de acuerdo a sus habilidades y conocimientos, por ello perciben un salario que por to general no satisface todas sus necesidades por diversas razones, ya sea de índole político-económicas del país y/o por el déficit presupuestario que agobia a las entidades públicas. Esta situación es de gran peso en la motivación, aunado a que no se cuenta con un sistema de evaluación de la productividad que al mismo tiempo sirva de recompensa por haber cumplido con las actividades asignadas. Se enfatiza que, si la 


\section{CIENCIAMATRIA}

Revista Interdisciplinaria de Humanidades, Educación, Ciencia y Tecnología

Año VII. Vol. VII. N¹. Edición Especial. 2021

Hecho el depósito de ley: pp201602FA4721

ISSN-L: 2542-3029; ISSN: 2610-802X

Universidad Nacional Experimental Francisco de Miranda (UNEFM). Santa Ana de Coro. Venezuela

Willians Antonio Guerrero-Castro; Rously Eedyah Atencio-González; Jesús Antonio Pirona

prestación de servicio de los empleados es cancelada por un sueldo asignado, no deja de ser de gran relevancia el reconocimiento por haberlo realizado en tiempo record y con calidad en sus resultados, mejor aún, si éstas cumplen con los objetivos establecidos en la planeación.

Las empresas están abocadas a incorporar nuevas estrategias para ser competitivas y sus talentos humanos a tener los conocimientos, habilidades y destrezas que les permitan mantenerse en el mercado de trabajo y a desenvolverse en forma competente en los actuales procesos productivos. Estos procesos, cada vez más exigentes, se caracterizan por la búsqueda de la creatividad y deben ser gestionados con inteligencia por redes de trabajo que se constituyen al interior de la empresa, conformados de funciones polivalentes y que apuntan a una producción diversificada.

La evaluación del desempeño es un instrumento; que constituye uno de los subsistemas de la gestión de recursos humanos que permite que las organizaciones se vuelvan altamente productivas, ya que a través de éste se logran resultados claves para su éxito. Asimismo, el proceso de evaluar permite medir el grado en que cada trabajador mantiene su idoneidad y cumple los objetivos del cargo o puesto de trabajo que desempeña (eficacia) así como la forma en que utiliza sus recursos para lograr dichos objetivos (eficiencia). Al referirse a la evaluación de las competencias laborales de un trabajador, se refiere al ¿Qué sabe hacer?, ¿Cuánto sabe?, ¿Por qué lo sabe?, ¿Cómo lo aplica? y ¿Cómo se desempeña en su puesto de trabajo? Con todo esto, además, se toma en cuenta en qué medida y tiempo en que lo hace. Por medio de la evaluación del desempeño se puede dar cuenta de las competencias, que se poseen contra las requeridas para ejecutar adecuadamente las funciones y tareas encomendadas para el trabajo y, en consecuencia, dan como resultado las necesidades de mejora, corrección y capacitación del personal.

El desempeño de los miembros de las organizaciones constituye una de las piedras angulares para lograr la efectividad y alcanzar el éxito de estas, razón por la cual hay un 


\section{CIENCIAMATRIA}

Revista Interdisciplinaria de Humanidades, Educación, Ciencia y Tecnología

Año VII. Vol. VII. N¹. Edición Especial. 2021

Hecho el depósito de ley: pp201602FA4721

ISSN-L: 2542-3029; ISSN: 2610-802X

Universidad Nacional Experimental Francisco de Miranda (UNEFM). Santa Ana de Coro. Venezuela

Willians Antonio Guerrero-Castro; Rously Eedyah Atencio-González; Jesús Antonio Pirona

constante interés de las entidades por mejorar los sistemas de gestión en que se sustenta el mismo, Montejo (2009:4) define desempeño como aquellas acciones o comportamientos observados en los empleados que son relevantes para los objetivos de la organización y que pueden ser medidos en términos de las competencias de cada individuo y su nivel de contribución a la empresa. Algunos investigadores argumentan que la definición de desempeño debe ser completada con la descripción de lo que se espera de los empleados, además de una continua orientación hacia el desempeño efectivo. Por otra parte, Alles (2005:18) describe el desempeño laboral como la relación entre el trabajo realizado y los resultados obtenidos por el mismo en beneficio de la organización, no depende únicamente de quien realiza las labores y las funciones que corresponden a un determinado trabajo, sino que además está condicionado a factores externos a su persona y que corresponden al clima o ambiente dentro del cual se desenvuelve y a la estructura formal en la cual están definidas tales funciones.

Para, Chiavenato (1999:125), la evaluación del desempeño permite implementar nuevas políticas de compensación, mejora el desempeño, ayuda a tomar decisiones de ascensos o de ubicación, también permite evaluar si existe la necesidad de volver a capacitar, detectar errores en el diseño del puesto y ayuda a observar si existen problemas personales que afecten a la persona en el desempeño del cargo. Por tal razón, una evaluación del desempeño trae beneficios tanto al evaluador como al evaluado.

Las ventajas y desventajas de cada método se visualizan a continuación: 


\section{Tabla 1.}

Ventajas y desventajas de los modelos.

\begin{tabular}{|c|c|c|}
\hline & Ventajas & Desventajas \\
\hline $\begin{array}{l}\text { Métodos } \\
\text { características }\end{array}$ & $\begin{array}{l}\text { De fácil y rápido diseño y, por lo } \\
\text { tanto, de menor coste. } \\
\text { Fáciles de usar. }\end{array}$ & $\begin{array}{l}\text { No son tan útiles para dar } \\
\text { devolución a los empleados y el } \\
\text { margen de error es mayor }\end{array}$ \\
\hline $\begin{array}{ll}\text { Métodos } & \text { de } \\
\text { comportamiento }\end{array}$ & $\begin{array}{l}\text { Se pueden definir estándares de } \\
\text { desempeño que son fácilmente } \\
\text { aceptados por jefes y } \\
\text { subordinados. Son muy útiles } \\
\text { para dar devolución de la } \\
\text { evaluación. }\end{array}$ & $\begin{array}{l}\text { El desarrollo puede requerir } \\
\text { mucho tiempo y es costoso. }\end{array}$ \\
\hline $\begin{array}{l}\text { Métodos } \\
\text { resultados }\end{array}$ & $\begin{array}{l}\text { Evitan la subjetividad y son } \\
\text { fácilmente aceptados por jefes y } \\
\text { subordinados. Relacionan el } \\
\text { desempeño de las personas con la } \\
\text { organización. Fomentan los } \\
\text { objetivos compartidos. }\end{array}$ & $\begin{array}{l}\text { El desarrollo puede requerir } \\
\text { mucho tiempo y pueden fomentar } \\
\text { en los empleados un enfoque de } \\
\text { corto plazo. }\end{array}$ \\
\hline
\end{tabular}

Fuente: Alles (2005).

Por todo lo tratado anteriormente, se planteó como objetivo general diagnosticar la situación actual en cuanto a la evaluación del desempeño llevada a cabo al personal administrativo de las instituciones universitarias.

\section{METODOLOGÍA}

La presente investigación desde el paradigma cuantitativo, se desarrolló dentro de los parámetros de un tipo de investigación de campo que, consiste en la recolección de datos directamente de la realidad donde ocurren los hechos sin manipular o controlar variables, en su ambiente natural, con la intención de recolectar los datos reales del proceso y resultados de las evaluaciones de desempeño y el diseño de investigación de tipo proyecto factible que, según Pallela \& Martins (2012), la define como el proceso de 


\section{CIENCIAMATRIA}

Revista Interdisciplinaria de Humanidades, Educación, Ciencia y Tecnología

Año VII. Vol. VII. N¹. Edición Especial. 2021

Hecho el depósito de ley: pp201602FA4721

ISSN-L: 2542-3029; ISSN: 2610-802X

Universidad Nacional Experimental Francisco de Miranda (UNEFM). Santa Ana de Coro. Venezuela

Willians Antonio Guerrero-Castro; Rously Eedyah Atencio-González; Jesús Antonio Pirona

elaborar una propuesta viable destinada a atender las necesidades específicas determinadas a partir de una base diagnóstica.

Los estudios descriptivos según Sampieri (1998:33), miden de manera más independiente los conceptos o variables a los que se refiere, pueden ofrecer la posibilidad de predicciones incipientes, aunque sean rudimentarias. Con relación a los instrumentos, Palella \& Martins (2012), señalan que un instrumento de recolección de datos es en principio, cualquier recurso del cual pueda valerse el investigador para acercarse a los fenómenos y extraer de ellos la información. En este sentido, para la investigación se utilizó un cuestionario.

\section{RESULTADOS}

A continuación, se muestran algunos resultados obtenidos de la encuesta aplicada a un grupo de trabajadores universitarios.

\section{Tabla 2.}

Uso de la evaluación del desempeño.

Indicador: Conocimientos Técnicos.

\begin{tabular}{|c|c|c|c|c|c|c|c|c|c|c|c|}
\hline \multirow[t]{2}{*}{$\mathbf{N}^{\circ}$} & \multirow[t]{2}{*}{ Ítems } & \multicolumn{2}{|c|}{ Siempre } & \multicolumn{2}{|c|}{$\begin{array}{c}\text { Casi } \\
\text { Siempre }\end{array}$} & \multicolumn{2}{|c|}{$\begin{array}{c}\text { Algunas } \\
\text { Veces }\end{array}$} & \multicolumn{2}{|c|}{ Casi Nunca } & \multicolumn{2}{|c|}{ Nunca } \\
\hline & & $\mathrm{F}$ & $\%$ & $F$ & $\%$ & $\mathrm{~F}$ & $\%$ & $F$ & $\%$ & $\bar{F}$ & $\%$ \\
\hline 1 & $\begin{array}{l}\text { La evaluación del desempeño es } \\
\text { un proceso positivo para la } \\
\text { organización. }\end{array}$ & 59 & 92,19 & 0 & 0,00 & 3 & 4,69 & 2 & 3,13 & 0 & 0,00 \\
\hline 2 & $\begin{array}{l}\text { La evaluación del desempeño } \\
\text { por competencias laborales es } \\
\text { conocida por el trabajador. }\end{array}$ & 28 & 43,75 & 12 & 18,75 & 23 & 35,94 & 1 & 1,56 & 0 & 0,00 \\
\hline
\end{tabular}

Fuente: Cuestionario aplicado al personal administrativo universitario. 


\section{CIENCIAMATRIA}

Revista Interdisciplinaria de Humanidades, Educación, Ciencia y Tecnología

Año VII. Vol. VII. N¹. Edición Especial. 2021

Hecho el depósito de ley: pp201602FA4721

ISSN-L: 2542-3029; ISSN: 2610-802X

Universidad Nacional Experimental Francisco de Miranda (UNEFM). Santa Ana de Coro. Venezuela

Willians Antonio Guerrero-Castro; Rously Eedyah Atencio-González; Jesús Antonio Pirona

La tabla 2 de la dimensión uso de la evaluación del desempeño, correspondiente al indicador conocimientos técnicos en su ítem $\mathrm{N}^{\circ} 1$, presenta que el $92,19 \%$ de los trabajadores administrativos encuestados afirman que la evaluación del desempeño es un proceso positivo para la universidad. No obstante, el 4,69\% de los trabajadores del instituto opina que solo algunas veces se puede valorar como positivo las evaluaciones de desempeño debido a que algunas de ellas no son aplicadas con objetividad o sus métodos están obsoletos.

En contraste a estas opiniones, el 3,13\% cree que casi nunca es positivas la aplicación de estas mediciones de la productividad. Asimismo, Alles (2005:27), refiere que "una evaluación de desempeño debe realizarse siempre con relación al perfil del puesto; sólo se podrá decir que una persona se desempeña bien o mal en relación con algo, en este ese algo es el puesto que ocupa". Es decir, que la utilización de la evaluación del desempeño en la organización es una herramienta imprescindible que no sólo aporta información sobre el comportamiento del empleado, sino también sobre su funcionamiento en general de forma que se satisfacen las expectativas y cómo hacer para mejorar los resultados. A continuación, se presenta gráficamente la contrastación de las respuestas obtenidas. 


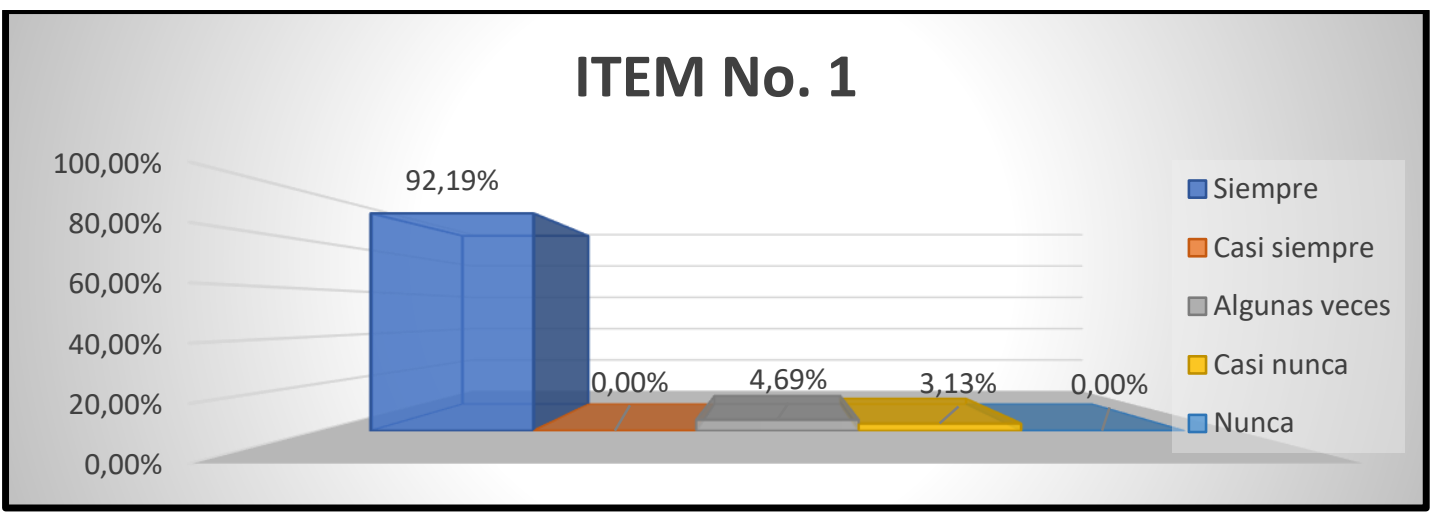

\section{Grafica 1.}

Fuente: Cuestionario aplicado al personal administrativo universitario.

Al respecto de las respuestas obtenidas en el ítem $\mathrm{N}^{\circ} 2$ de la misma dimensión, uso de la evaluación del desempeño, correspondiente al indicador conocimientos técnicos, se refleja que el $43,75 \%$ aprecia que siempre las evaluaciones del desempeño por competencias son conocidas por el trabajador, ya que aunado a la aplicación de estos instrumentos se evalúan los objetivos de desempeño individual ya establecidos durante el período evaluado (regularmente semestrales), lo cual ayuda a medir el cumplimiento de las metas trazadas en la realización del trabajo ejercido.

En contraste, el 35,94\% de los encuestados creen que solo algunas veces son conocidos estos métodos de evaluación del desempeño para los trabajadores, pues se mantienen en una posición en la cual esperan que se realice una verdadera evaluación del personal sin sesgos de ninguna naturaleza, entre ellos la objetividad como principal aspecto, mientras que el $18,75 \%$ de los administrativos opinan que casi siempre se conocen estas evaluaciones. 


\section{CIENCIAMATRIA}

Revista Interdisciplinaria de Humanidades, Educación, Ciencia y Tecnología

Año VII. Vol. VII. N¹. Edición Especial. 2021

Hecho el depósito de ley: pp201602FA4721

ISSN-L: 2542-3029; ISSN: 2610-802X

Universidad Nacional Experimental Francisco de Miranda (UNEFM). Santa Ana de Coro. Venezuela

Willians Antonio Guerrero-Castro; Rously Eedyah Atencio-González; Jesús Antonio Pirona

\section{ITEM No. 2}

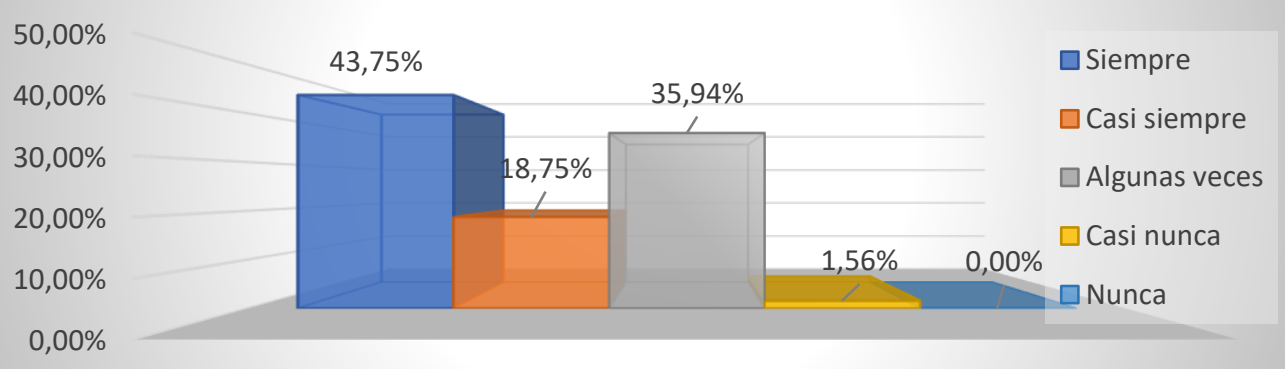

\section{Grafica 2.}

Fuente: Cuestionario aplicado al personal administrativo universitario.

\section{Tabla 3.}

Administración del desempeño por competencias. Indicador: Actitudes.

\begin{tabular}{|c|c|c|c|c|c|c|c|c|c|c|c|}
\hline \multirow[t]{2}{*}{$\mathbf{N}^{\circ}$} & \multirow[t]{2}{*}{ Ítems } & \multicolumn{2}{|c|}{ Siempre } & \multicolumn{2}{|c|}{$\begin{array}{c}\text { Casi } \\
\text { Siempre }\end{array}$} & \multicolumn{2}{|c|}{$\begin{array}{l}\text { Algunas } \\
\text { Veces }\end{array}$} & \multicolumn{2}{|c|}{ Casi Nunca } & \multicolumn{2}{|c|}{ Nunca } \\
\hline & & $\bar{F}$ & $\%$ & $\bar{F}$ & $\%$ & $\mathbf{F}$ & $\%$ & $\mathbf{F}$ & $\%$ & $\mathbf{F}$ & $\%$ \\
\hline 3 & $\begin{array}{|lrr|}\text { La evaluación } & \text { del desempeño } \\
\text { puede verse afectada } & \text { por } \\
\text { elementos } & \text { subjetivos } & \text { del } \\
\text { evaluador tales como problemas } \\
\text { personales, prejuicios, entre } \\
\text { otros. }\end{array}$ & 54 & 84,38 & 3 & 4,69 & 2 & 3,13 & 3 & 4,69 & 2 & 3,13 \\
\hline 4 & $\begin{array}{l}\text { Los aspectos que se miden en el } \\
\text { modelo de evaluación del } \\
\text { desempeño por objetivos están } \\
\text { acordes a las funciones que tiene } \\
\text { cada puesto de trabajo. }\end{array}$ & 9 & 14,06 & 52 & 81,25 & 3 & 4,69 & 0 & 0,00 & 0 & 0,00 \\
\hline
\end{tabular}

Fuente: Cuestionario aplicado al personal administrativo universitario. 


\section{CIENCIAMATRIA}

Revista Interdisciplinaria de Humanidades, Educación, Ciencia y Tecnología

Año VII. Vol. VII. N¹. Edición Especial. 2021

Hecho el depósito de ley: pp201602FA4721

ISSN-L: 2542-3029; ISSN: 2610-802X

Universidad Nacional Experimental Francisco de Miranda (UNEFM). Santa Ana de Coro. Venezuela

Willians Antonio Guerrero-Castro; Rously Eedyah Atencio-González; Jesús Antonio Pirona

Las alternativas de respuestas de la tabla 3 , del ítem $N^{\circ} 3$, de la dimensión administración del desempeño por competencias, indicador actitudes, el 84,38\% opinaron que siempre la evaluación del desempeño puede ser impactada, positiva o negativamente, por elementos intrínsecos del evaluador, ya que en muchas circunstancias quienes son responsables de llevar a cabo esta medición no lo hacen con ética, objetividad, profesionalismo e imparcialidad, lo cual perjudica directamente al trabajador, siendo esto una conducta del antivalor de la que no deben estar presente en la administración pública nacional, por ser esta violatoria y contraria al derecho constitucional que nos asiste como trabajadores.

Por esta razón se favorece la investigación, con el fin de darle a conocer la importancia de esta valiosa herramienta de medición y su influencia en la toma de decisiones al momento de evaluar la productividad al recurso más importante de toda organización como lo es el talento humano.

En contraste, el 4,69\% considera que casi nunca estos aspectos impactan en las evaluaciones. Por su parte Chiavenato (1999:198), la define la evaluación del desempeño como "el proceso de revisar la actividad productiva del pasado para evaluar la contribución que el trabajador hace para que se logren los objetivos del sistema administrativo". Esto es parte de administrar los talentos humanos, para ello en las organizaciones debería existir un sistema metódico, objetivo eficiente de evaluación del desempeño, siendo este imprescindible para generar necesidades de perfeccionamiento continúo en los empleados, en el cual los mismos, se someten consciente y periódicamente a un proceso de evaluación de su desempeño, en aras de fortalecer sus potencialidades. 


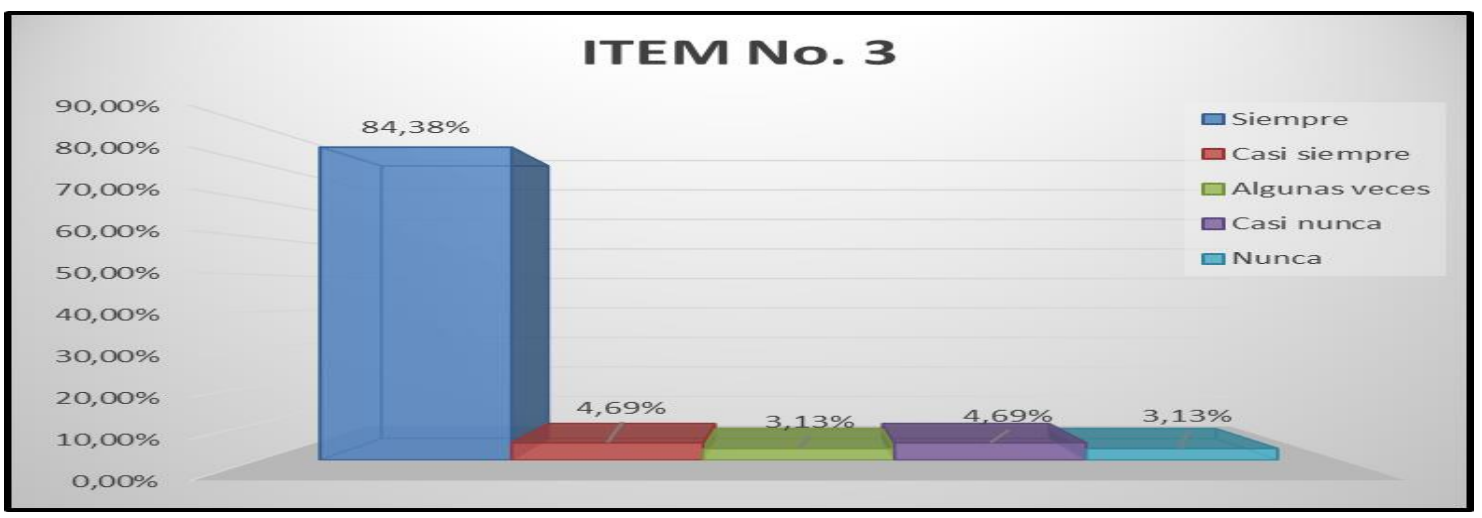

\section{Gráfica 3.}

Fuente: Cuestionario aplicado al personal administrativo universitario.

Con relación al ítem $\mathrm{N}^{\circ} 4$ de la misma dimensionalidad, administración del desempeño por competencias, indicador actitudes, el $81,25 \%$ de los participantes afirman que casi siempre son considerados los aspectos que se miden en la evaluación de desempeño por competencias están acordes con las funciones de los puestos de trabajo por cuanto son analizados por el perfil de las actividades que ejercen cada uno de los trabajadores administrativos. Así mismo, en alusión a estas respuestas, el 14,06 \% concluyó que siempre son evaluados los empleados acordes a los puestos que ocupan y las funciones que ejercen. A continuación, se detalla gráficamente las respuestas obtenidas en este ítem. 


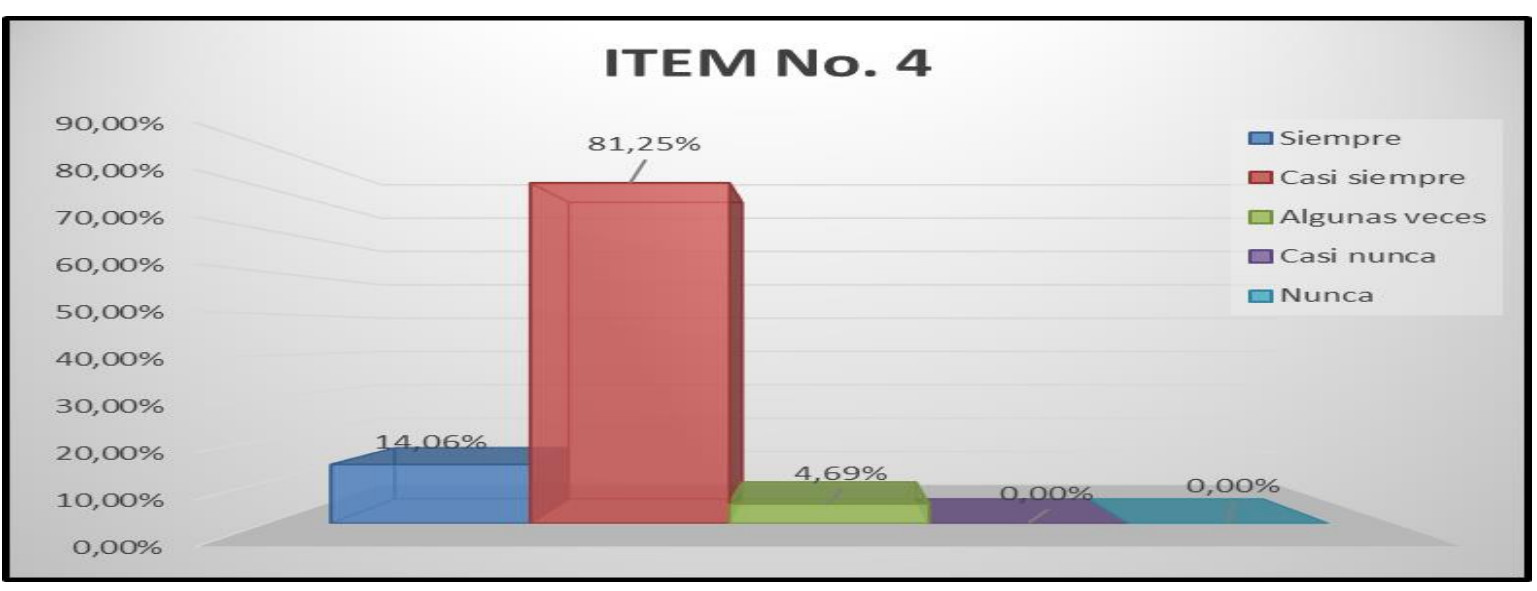

\section{Grafica 4.}

Fuente: Cuestionario aplicado al personal administrativo universitario.

\section{CONCLUSIONES}

Partiendo de los resultados analizados, se hace necesario aplicar un instrumento de evaluación basado en competencias laborales que sustituya el modelo aplicado hasta el momento, basado en resultados por objetivos de desempeño individual. En la administración pública nacional han convivido con persistentes pautas regidas por modelos burocráticos de gestión, que no han tenido en cuenta la importancia del factor humano en el desenvolvimiento de la administración, aspecto que con gran frecuencia ha paralizado el proceso de cambio de las estructuras organizativas del Estado y las formas de organización del trabajo, atrofiando el funcionamiento de las mismas en detrimento de la eficiencia y eficacia del órgano público. Por esta razón, concretar nuevos modelos de gestión de los recursos humanos del Estado es un reto significativo. 


\section{REFERENCIAS}

Alles, M. (2005) Desempeño por Competencias. Evaluación de $360^{\circ}$. 2da Edición. Buenos Aires, Argentina.

Asamblea Nacional de República Bolivariana de Venezuela. (2002) Ley del Estatuto de la Función Pública. Gaceta Oficial No. 37.522 de fecha 06 de septiembre de 2002. Venezuela.

Centro Latinoamericano de Administración para el Desarrollo NU (DESA) Naciones Unidas Departamento de Asuntos Económicos y Sociales (1972) Carta Iberoamericana de la Función Pública (2003). Recuperado de: https://n9.cl/ine68

Chiavenato, I. (1999) Administración de Recursos Humanos. Quinta edición. México, D.F. McGraw Hill/Interamericana Editores, S.A. de C.V.

Freites, R. (2007). Sistema de Evaluación del Desempeño para el personal administrativo del "Instituto Pedagógico de Miranda José Manuel Siso Martínez". Trabajo de Grado para optar al Título Académico de "Bachelor of Business Administration in Administrative Services".

Hernández-Sampieri, R., Fernández-Collado, C., \& Baptista-lucio, P. (2006) Metodología de la investigación. 4Ta Edición. Editorial: MCGRAWHILL. México.

Palella Stracuzzi, S. \& Martins Pestana, F. (2012). Metodología de la investigación cuantitativa. Fondo editorial de la Universidad Pedagógica Libertador. Caracas, Venezuela.

Pérez-Montejo, A. (2009) Evaluación del Desempeño LaboralUPIICSA, 17(7),50-51. Recuperado de: http://148.204.210.204/revistaupiicsa/50-51/50-51-2.pdf

Rodríguez-Serrano, J. (2004) El modelo de gestión de recursos humanos un enfoque tradicionalmente nuevo sobre gestión de personas. Editorial UOC. Universidad Oberta de Catalunya. 
Revista Interdisciplinaria de Humanidades, Educación, Ciencia y Tecnología

Año VII. Vol. VII. N¹. Edición Especial. 2021

Hecho el depósito de ley: pp201602FA4721

ISSN-L: 2542-3029; ISSN: 2610-802X

Universidad Nacional Experimental Francisco de Miranda (UNEFM). Santa Ana de Coro. Venezuela

Willians Antonio Guerrero-Castro; Rously Eedyah Atencio-González; Jesús Antonio Pirona

Sánchez-Henríquez, J. \&. Calderón-Calderón, V. (2012) Diseño del proceso de evaluación del desempeño del personal y las principales tendencias que afectan su auditoría. Pensamiento \& Gestión, 32, 54-82. Recuperado de: http://www.scielo.org.co/pdf/pege/n32/n32a04.pdf

C2021 por los autores. Este artículo es de acceso abierto y distribuido según los términos y condiciones de la licencia Creative Commons Atribución-NoComercial-Compartirlgual 4.0 Internacional (CC BY-NC-SA 4.0) (https://creativecommons.org/licenses/by-nc-sa/4.0/). 